\title{
Surgery for acute type A dissection using total arch replacement combined with stented elephant trunk implantation: Preservation of autologous brachiocephalic vessels
}

\author{
Jun-Ming Zhu, MD, ${ }^{\mathrm{a}}$ Rui-Dong Qi, MD, ${ }^{\mathrm{a}}$ Lei Chen, MD,${ }^{\mathrm{a}}$ Wei Liu, MD, ${ }^{\mathrm{a}}$ Cheng-Nan Li, MD, \\ Zhan-Ming Fan, MD, ${ }^{b}$ and Li-Zhong Sun, MD ${ }^{a}$
}

\begin{abstract}
Objective: Various techniques have been introduced to treat acute type A dissection during aortic arch reconstruction. We retrospectively reviewed our experience of total arch replacement, with implantation of a stented elephant trunk, using preservation of autologous brachiocephalic vessels in patients with acute type A dissection.
\end{abstract}

Methods: Between August 2011 and April 2013, 20 patients (16 men, 4 women; mean age, $45 \pm 10$ years, range, 24-62 years) with acute type A dissection underwent total arch replacement combined with stented elephant trunk implantation, using preservation of autologous brachiocephalic vessels under hypothermic cardiopulmonary bypass with selective antegrade cerebral perfusion.

Results: No in-hospital deaths occurred. A transient neurologic deficit occurred in 1 patient, who ultimately required tracheotomy. Reoperation was indicated in 1 patient for bleeding. All patients survived and were discharged. During the mean follow-up period of $26 \pm 7$ months, 1 patient underwent thoracoabdominal aortic replacement, and 1 patient was lost to follow up. The patency of the anastomotic site between the left subclavian artery and the left common carotid artery was confirmed on computed tomography scanning.

Conclusions: This technique simplified hemostasis and anastomosis, reduced the size of the residual aortic wall, and preserved the autologous brachiocephalic vessels, yielding satisfactory surgical results. This technique is an alternative approach for suitable patients with acute type A dissection. However, outcomes are preliminary, and long-term follow up is required. ( $\mathrm{J}$ Thorac Cardiovasc Surg 2015;150:101-5)

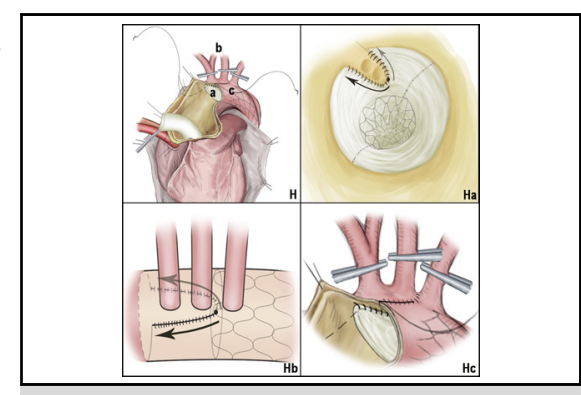

View of the neoaortic arch from the inside, the greater curvature side and the anterior.

\section{Central Message}

This technique simplified hemostasis and anastomosis, reduced the size of the residual aortic wall, and preserved the autologous brachiocephalic vessels, yielding satisfactory surgical results

\section{Perspective}

The technique of preservation of the autologous brachiocephalic vessels for total arch replacement, with implantation of a stented elephant trunk, simplifies hemostasis and anastomosis, decreases the risk of aneurysmal dilatation of the residual aortic wall, and has the advantage of long-term patency. Satisfactory surgical results and follow-up outcomes are achieved in this study. Our experience demonstrates that this technique is an alternative approach for suitable patients with acute type A dissection.

See Editorial Commentary page 106
Acute type A dissection is a lethal disease associated with high morbidity and mortality. Owing to advances in surgical techniques, anesthesia, and cardiopulmonary bypass (CPB), more surgeons favor extended aortic arch replacements in patients with acute type A dissection. To improve late

\footnotetext{
From the Beijing Aortic Disease Center, ${ }^{\mathrm{a}}$ and Department of Radiology, ${ }^{\mathrm{b}}$ Beijing Institute of Heart, Lung and Blood Vessel Diseases \& Beijing Anzhen Hospital, Capital Medical University, Beijing, China.

Received for publication Dec 5, 2014; revisions received Feb 16, 2015; accepted for publication March 2, 2015; available ahead of print April 8, 2015.

Address for reprints: Li-Zhong Sun, MD, Beijing Aortic Disease Center, Beijing Anzhen Hospital, Capital Medical University, 2 Anzhen Rd, Beijing 100029, China (E-mail: lizhongsun@outlook.com).

$0022-5223 / \$ 36.00$

Copyright (C) 2015 by The American Association for Thoracic Surgery http://dx.doi.org/10.1016/j.jtcvs.2015.03.002
}

surgical outcome, total aortic replacement is recommended as the initial surgical approach for acute type A dissection, by us and others. ${ }^{1-4}$ Several surgical approaches, such as the separate graft, ${ }^{5,6}$ en bloc, ${ }^{5}$ hybrid, ${ }^{7}$ and branched stent graft placement $^{8}$ techniques have been adopted for aortic arch reconstruction. We reviewed our experience of surgery for acute type A dissection using total arch replacement, combined with stented elephant trunk implantation, with preservation of the autologous brachiocephalic vessels.

\author{
METHODS \\ Patients \\ Between August 2011 and April 2013, 20 patients (16 men, 4 women; \\ mean age, $45 \pm 10$ years, range, 24-62 years) with acute type A dissection
}




$$
\begin{aligned}
& \text { Abbreviations and Acronyms } \\
& \begin{aligned}
\text { CPB } & =\text { cardiopulmonary bypass } \\
\text { IA } & =\text { innominate artery } \\
\text { LCCA } & =\text { left common carotid artery } \\
\text { LSCA } & =\text { left subclavian artery } \\
\text { SACP } & =\text { selective antegrade cerebral perfusion }
\end{aligned}
\end{aligned}
$$

in our center underwent total arch replacement, combined with stented elephant trunk implantation, using preservation of autologous brachiocephalic vessels under hypothermic CPB with selective antegrade cerebral perfusion (SACP). This technique was approved by the institutional review board of Capital Medical University. Surgery was performed within 2 weeks after the onset of pain. Patients with acute type A dissection underwent preoperative computed tomography and echocardiography. Three patients had Marfan syndrome; 18 patients had a history of hypertension; and 1 patient had a previous Bentall procedure. One patient had a failed thoracic endovascular aortic repair. Preoperative comorbidities were common (Table 1).

The tear site was located in the ascending aorta in 8 patients, at the transverse arch in 2 patients, and in the proximal descending thoracic aorta in 10 patients. The dissection extended into the descending thoracic aorta in 2 cases, the abdominal aorta in 1 patient, and the iliac artery in 17 patients.

\section{Surgical Technique}

The right axillary artery was exposed through a right subclavicular incision. A median sternotomy was performed in all patients. The brachiocephalic vessels and the transverse arch were dissected and exposed (Figure 1,A). The key step in the end-to-side anastomosis between the 2 vessels was dissection of the left subclavian artery (LSCA) and the left common carotid artery (LCCA). To aid this procedure, the left sternocleidomastoid muscle and other cervical muscle groups were partially transected.

After the LCCA was fully dissected, the LSCA was easily separated behind the left side of the LCCA. The extent of dissection ranged from the transverse arch to the origin of the left vertebral artery. During dissection, care was taken to avoid injury to the thoracic duct; if necessary, ligation of the thoracic duct was performed.

Cannulation of the right axillary artery was used for CPB and SACP; $\mathrm{CPB}$ was instituted by cannulation of the right axillary artery and the right atrium. The left heart was vented via the right inferior pulmonary vein. Cardioplegia was performed directly within the coronary ostia with intermittent antegrade perfusion of cold-blood cardioplegic solution. Cerebral circulation in the left hemisphere was considered adequate, using unilateral SACP, if the left radial arterial pressure was $20 \mathrm{~mm} \mathrm{Hg}$ and back-bleeding via the LCCA was seen.

The concomitant procedure (aortic root procedure or coronary artery bypass grafting) was performed during the cooling phase (Figure 1, B). After the nasopharyngeal temperature reached $25^{\circ} \mathrm{C}$, the brachiocephalic vessels were clamped. Circulatory arrest was instituted, and the brain was perfused at approximately 5 to $10 \mathrm{ml} / \mathrm{kg}$ per minute, using SACP.

The ascending aorta was unclamped and opened, and the anterior wall of the aortic arch was incised longitudinally up to the origin of the LCCA (Figure 1,C). The brachiocephalic vessels were inspected closely, and if they were positioned such that they would not be involved in aortic dissection, then this technique was performed. The residual aortic arch wall containing the innominate artery (IA) and the LCCA was incised approximately $0.5 \mathrm{~cm}$ distal to the origin of the IA and LCCA.

The surgical stent-graft (Cronus, Microport, Shanghai, China), described in detail in our previous study, ${ }^{9-11}$ was implanted into the true lumen of the distal aorta. The proximal edge of the metallic stent (not the stent-free sewing edge) was positioned between the origin of the LCCA and the LSCA
(Figure 1,D). The stent-free sewing edge was pulled, and the spiralshaped Dacron graft could be straightened to approximately $3.0 \mathrm{~cm}$ in length (Figure 1,E). The straightened prosthetic graft was trimmed to match the estimated shape of the aortic arch wall containing the IA and the LCCA (Figure 1, F). The ends of the IA and the LCCA were exposed clearly.

A double-ended 4-0 Prolene (polypropylene; Ethicon, Inc, Somerville, $\mathrm{NJ}$ ) suture was used to sew, starting within the straightened prosthetic graft between the level of the LSCA and LCCA origin. The first stitch (shown by the red circle in Figure 1, G [ "a" label] ) was passed through the straightened prosthetic graft and out through the autologous aortic wall tissue. The inner suture was run counterclockwise within the aortic arch, and the posterior trimmed stent-free sewing edge was sutured to the autologous aortic arch wall adjacent to the IA and LCCA origin using a continuous suture line (Figure 1, $\mathrm{G}$; Figure 2, $\mathrm{Ha}$ ).

After that step, the outer double-ended 4-0 Prolene (polypropylene; Ethicon, Inc, Somerville, NJ) suture was run clockwise outside the aortic arch (Figure 1, $H$; Figure 2, $H b$ ). The trimmed anterior stent-free sewing edge incorporated in its outside aortic wall was sutured to the aortic arch wall containing the IA and LCCA, using a running line (Figure 1, $H$; Figure 2, $H b$ ). Thus, the residual aortic arch wall and the trimmed stent-free sewing edge commonly formed a rounded opening (Figure 1, $H$; Figure 2, $H c$ ).

The distal end of a woven Dacron graft, which was anastomosed to the aortic root, was anastomosed to the distal aorta containing the residual aortic arch wall and the surgical graft, using open distal anastomosis (Figure 1,I). After the anastomosis was completed (Figure 1,J), the CPB was gradually restored to normal flow, and rewarming was started. After the LSCA was transected circumferentially, 0.5 to $1.0 \mathrm{~cm}$ distal to its origin (Figure $1, K$ ), the origin of the LSCA was sutured using a 5-0 Prolene (polypropylene; Ethicon, Inc, Somerville, NJ) suture. Finally, the LSCA was anastomosed to the LCCA in an end-to-side fashion, using 6-0 Prolene (polypropylene; Ethicon, Inc, Somerville, NJ) (Figure 1, L).

\section{RESULTS \\ Surgical Data}

Patients with acute type A dissection underwent total arch replacement combined with stented elephant trunk implantation, using preservation of autologous brachiocephalic vessels under hypothermic CPB with SACP. The CPB time was 117 to 246 (mean, $169 \pm 34$ ) minutes; aortic cross-clamp time was 46 to 153 (mean, $87 \pm 26$ ) minutes; and SACP time was 22 to 40 (mean, $29 \pm 5$ ) minutes. Concomitant procedures are summarized in Table 2.

\section{Morbidity and Mortality}

No in-hospital deaths occurred in our patient cohort. Seventeen patients required mechanical ventilation for $<24$ hours, and 2 patients for $<48$ hours. Only 1 patient with a transient neurologic deficit required prolonged mechanical ventilation for $>7$ days, ultimately requiring tracheotomy. Fortunately, he survived and was discharged. One patient required reoperation for bleeding. Continuous renal replacement therapy was not necessary in any patient.

\section{Follow-up}

One case was lost to follow-up during the mean follow-up period of $26 \pm 7$ (range, 17-37) months. One patient underwent thoracoabdominal aortic replacement 9 months after the initial surgery. He recovered well and was discharged 
TABLE 1. Clinical profiles of patients with acute type A dissection

\begin{tabular}{lc}
\hline \multicolumn{1}{c}{ Variables } & No. of patients $(\%)$ \\
\hline Age $(\mathrm{y})$ & $45 \pm 10$ \\
Male gender & $16(80)$ \\
Female gender & $4(20)$ \\
Hypertension & $18(90)$ \\
Marfan syndrome & $3(15)$ \\
Diabetes mellitus & $1(5)$ \\
Coronary artery disease & $2(10)$ \\
Renal dysfunction & $2(10)$ \\
Cerebral ischemia & $1(5)$ \\
Lower-extremity ischemia & $3(15)$ \\
\hline
\end{tabular}

Age is given as mean $\pm \mathrm{SD}$

and had a normal life thereafter. No injury occurred to the spinal cord, and visceral malperfusion was not observed.

Postoperative computed tomography scanning revealed patency of the anastomotic site between the LSCA and the LCCA (Figure 3). The aorta was normal in 2 patients who had aortic dissection limited to the descending aorta using this technique. In the remaining 18 patients, thrombus obliteration of the false lumen around the surgical graft was seen in $88.9 \%$ ( 16 of 18 ) of patients, partial thrombosis in $5.6 \%$ ( 1 of 18 ), and patency in 5.6\% (1 of 18). Complete thrombus formation extending to the diaphragm was observed in $13(72.2 \%)$ patients, at the diaphragm in 7 $(38.8 \%)$ patients, below the diaphragm in $6(33.3 \%)$ patients, and at the celiac trunk in $4(22.2 \%)$ patients.

\section{DISCUSSION}

In the surgical management of the lesions that involve the transverse aortic arch, various techniques and grafts for aortic arch replacement have been introduced: the Y-graft, ${ }^{12}$ trifurcated branched grafts, ${ }^{6}$ the 4-branched graft, ${ }^{5,13}$ open branched stent graft placement, ${ }^{8,14}$ the "en bloc" (island) technique, ${ }^{5,15,16}$ and prophylactic debranching of the supraaortic vessels. ${ }^{17}$ During aortic arch reconstruction, a separated graft technique presents several advantages compared with en bloc repair. ${ }^{5}$ For the latter, the serious risk is for postoperative aneurysmal dilatation of the residual aortic patch containing the brachiocephalic vessels. However, the en bloc technique has the advantage of long-term patency, owing to the preservation of the native brachiocephalic vessels.

To simplify total arch replacement, a double- or triplebranched stent graft was introduced to treat acute type A dissection. $^{8,14}$ However, postoperative graft shifting or kinking has been reported. ${ }^{18}$ These complications may lead to graft occlusion, aortic disruption, and organ malperfusion syndrome. ${ }^{18}$

Considering these challenges, a novel technique for total arch replacement was developed to treat type A dissection using the stented elephant trunk technique: (1) the stent-free sewing edge of the stented elephant trunk was grasped, using forceps, and the spiral-shaped Dacron graft $(1.0 \mathrm{~cm}$ in length) was straightened to approximately $3.0 \mathrm{~cm}$; (2) the native brachiocephalic vessels were preserved; (3) a residual aortic wall containing the IA and the LCCA was made as small as possible; and (4) an end-to-side anastomosis between the LSCA and the LCCA was performed. The indications for this procedure for type A dissection were: (1) total arch replacement was indicated for acute type A dissection. ${ }^{19}$ (2) The IA and LCCA have no involvement of aortic dissection, no aneurysm formation and no occlusion lesions. Owing to
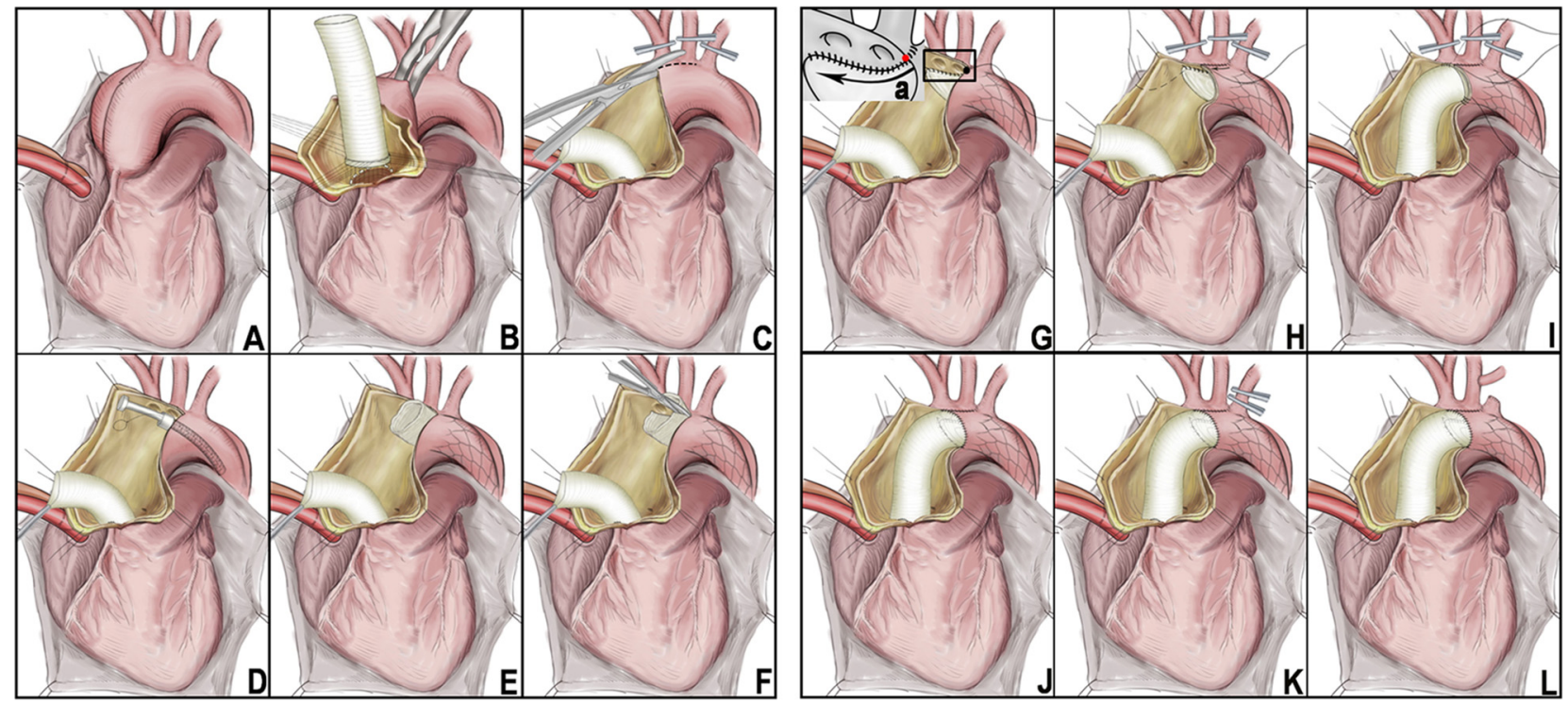

FIGURE 1. Technique of total arch replacement combined with stented elephant trunk, using preservation of autologous brachiocephalic vessels (see text for details). 


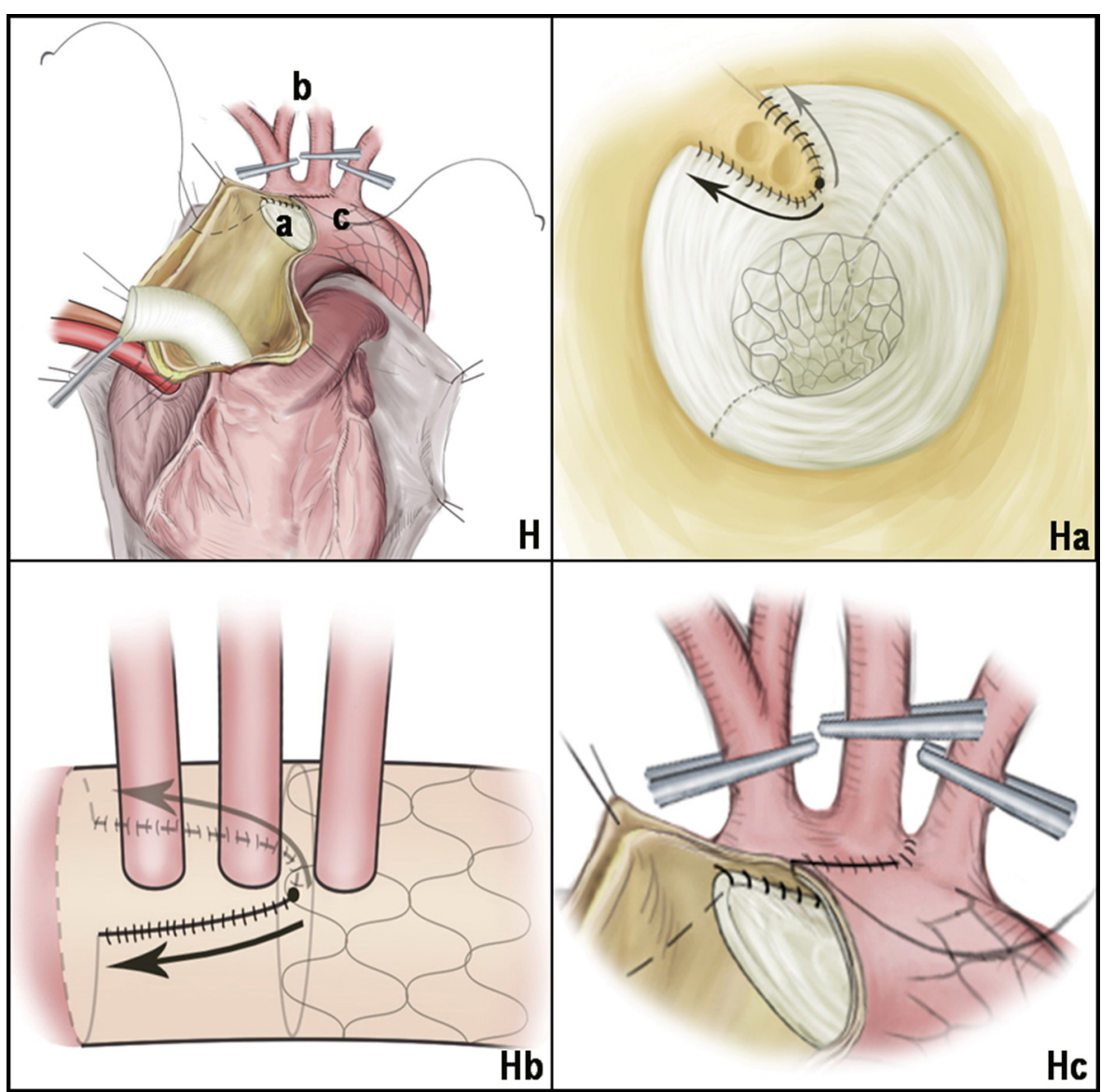

FIGURE 2. View of the neoaortic arch from the inside, the greater curvature side and the anterior. $H$, Implantation of the remaining aortic arch wall containing the IA and LCCA into the stent-free sewing edge (detailed description of Figure 1, $H$ ). $H a$, Inside view of the neoaortic arch. The site of the first stitch is indicated by the black circle. One end of the suture was run counterclockwise within the aortic arch. $H b$, The neoartic arch seen from the greater curvature side. The first stitch is shown by the black circle. The other end of the 4-0 Prolene (polypropylene; Ethicon, Inc, Somerville, NJ) suture was run clockwise outside the aortic arch. $H c$, The neoaortic arch from an anterior view; the remaining aortic arch wall and the trimmed stent-free sewing edge commonly form a distal rounded opening.

circumferential transection 0.5 to $1.0 \mathrm{~cm}$ distal to the LSCA origin, the resected distal portion was normal irrespective of whether the proximal stump of the LSCA was involved in aortic dissection. (3) No atherosclerotic or aneurysmal changes in the remaining aortic arch wall containing the IA and the LCCA. (4) Adequate cerebral circulation in the left hemisphere.

This technique has several advantages. First, the aortic wall was trimmed by approximately $0.5 \mathrm{~cm}$ adjacent to the origin of the IA and LCCA. The residual aortic wall was very small, which decreased the risk of postoperative

TABLE 2. Concomitant procedure

\begin{tabular}{lc}
\hline \multicolumn{1}{c}{ Variables } & No. of patients $(\%)$ \\
\hline Bentall procedure & $5(25)$ \\
Sinus of Valsalva reconstruction & $4(20)$ \\
Aortic valve plasty & $5(25)$ \\
Mitral valve replacement & $1(5)$ \\
\hline
\end{tabular}

aneurysmal dilatation of the residual aortic wall. Second, preservation of the autologous brachiocephalic vessels resulted in long-term patency. Third, the anastomosed site was moved ahead of the origin of the LSCA, simplifying hemostasis and anastomosis.

Excellent surgical outcomes have been obtained using this technique. No in-hospital deaths occurred in this group. The mean SACP time (lower-body ischemic time) was $29 \pm 5$ minutes, which did not increase using this technique, as illustrated by the fact that no severe complications occurred, except 1 instance of a transient neurologic deficit. Follow-up computed tomography demonstrated good patency of anastomotic site between the LSCA and the LCCA. Thrombosis and remodeling of the aortic false lumen in the distal aorta were similar to those in our previous reports. ${ }^{1,20}$ Although this procedure was performed in 3 patients with Marfan syndrome, a separate graft technique was preferred for Marfan patients with type A dissection. 


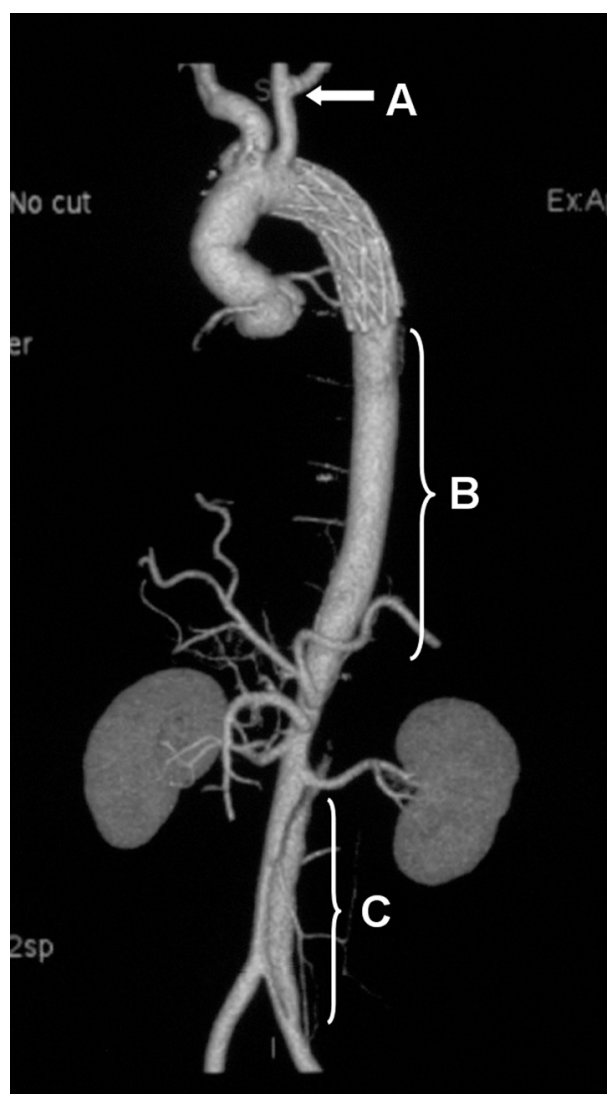

FIGURE 3. Three-dimensional computed tomography reconstruction of acute type A dissection using total arch replacement, combined with stented elephant trunk implantation, with preservation of autologous brachiocephalic vessels, 2 years after surgery. A, The patency of the anastomotic site between the LSCA and the LCCA. B, The descending thoracic aorta and upper abdominal aorta restored to normal after remodeling. C, The presence of the false lumen in the abdominal aorta below the left renal artery level.

\section{CONCLUSIONS}

Encouraging surgical results were obtained in patients with acute type A dissection, using this technique. Total arch replacement, combined with the stented elephant trunk technique, with preservation of autologous brachiocephalic vessels, was an alternative approach for suitable patients with acute type A dissection. However, our study was limited by the small sample size, and the results are preliminary. Close, long- term follow up is required.

\section{Conflict of Interest Statement}

Authors have nothing to disclose with regard to commercial support.

\section{References}

1. Sun LZ, Qi RD, Chang Q, Zhu JM, Liu YM, Yu CT, et al. Surgery for acute type A dissection using total arch replacement combined with stented elephant trunk implantation: experience with 107 patients. J Thorac Cardiovasc Surg. 2009; 138:1358-62.

2. Kazui T, Washiyama N, Muhammad BA, Terada H, Yamashita K, Takinami M, et al. Extended total arch replacement for acute type A aortic dissection: experience with seventy patients. J Thorac Cardiovasc Surg. 2000;119:558-65.

3. Hirotani T, Kameda T, Kumamoto T, Shirota S. Results of a total aortic arch replacement for an acute aortic arch dissection. J Thorac Cardiovasc Surg. 2000;120:686-91.

4. Ochiai Y, Imoto Y, Sakamoto M, Ueno Y, Sano T, Baba H, et al. Long-term effectiveness of total arch replacement for type A aortic dissection. Ann Thorac Surg. 2005;80:1297-302.

5. Di Eusanio M, Schepens MA, Morshuis WJ, Dossche KM, Kazui T, Ohkura K, et al. Separate grafts or en bloc anastomosis for arch vessels reimplantation to the aortic arch. Ann Thorac Surg. 2004;77:2021-8.

6. Spielvogel D, Etz CD, Silovitz D, Lansman SL, Griepp RB. Aortic arch replacement with a trifurcated graft. Ann Thorac Surg. 2007;83:S791-5; discussion S824-31.

7. Hofferberth SC, Newcomb AE, Yii MY, Yap KK, Boston RC, Nixon IK, et al. Hybrid proximal surgery plus adjunctive retrograde endovascular repair in acute DeBakey type I dissection: superior outcomes to conventional surgical repair. $J$ Thorac Cardiovasc Surg. 2013;145:349-54; discussion 354-5.

8. Chen LW, Dai XF, Lu L, Zhang GC, Cao H. Extensive primary repair of the thoracic aorta in acute type A aortic dissection by means of ascending aorta replacement combined with open placement of triple-branched stent graft: early results. Circulation. 2010;122:1373-8.

9. Liu ZG, Sun LZ, Chang Q, Zhu JM, Dong C, Yu CT, et al. Should the "elephant trunk" be skeletonized? Total arch replacement combined with stented elephant trunk implantation for Stanford type A aortic dissection. J Thorac Cardiovasc Surg. 2006;131:107-13.

10. Sun L, Qi R, Chang Q, Zhu J, Liu Y, Yu C, et al. Surgery for Marfan patients with acute type A dissection using a stented elephant trunk procedure. Ann Thorac Surg. 2008;86:1821-5.

11. Sun LZ, Qi RD, Chang Q, Zhu JM, Liu YM, Yu CT, et al. Is total arch replacement combined with stented elephant trunk implantation justified for patients with chronic Stanford type A aortic dissection? J Thorac Cardiovasc Surg. 2009; 138:892-6

12. LeMaire SA, Price MD, Parenti JL, Johnson ML, Lay AD, Preventza O, et al Early outcomes after aortic arch replacement by using the Y-graft technique. Ann Thorac Surg. 2011;91:700-7; discussion 707-8.

13. Kazui T, Washiyama N, Muhammad BA, Terada H, Yamashita K, Takinami M, et al. Total arch replacement using aortic arch branched grafts with the aid of antegrade selective cerebral perfusion. Ann Thorac Surg. 2000;70:3-8; discussion 8-9.

14. Shimamura K, Kuratani T, Matsumiya G, Shirakawa Y, Takeuchi M, Takano H, et al. Hybrid endovascular aortic arch repair using branched endoprosthesis: the second-generation "branched" open stent-grafting technique. J Thorac Cardiovasc Surg. 2009; 138:46-52; discussion 52-3.

15. Shrestha M, Martens A, Behrendt S, Maeding I, Koigeldiyev N, Haverich A. Is the branched graft technique better than the en bloc technique for total aortic arch replacement? Eur J Cardiothorac Surg. 2014;45:181-7.

16. Pearce CW, Weichert RF III, del Real RE. Aneurysms of aortic arch. Simplified technique for excision and prosthetic replacement. J Thorac Cardiovasc Surg. 1969;58:886-90.

17. Glauber M, Murzi M, Farneti P, Bevilacqua S, Mariani M, Tognarelli A, et al Aortic arch replacement with prophylactic aortic arch debranching during type A acute aortic dissection repair: initial experience with 23 patients. Eur J Cardiothorac Surg. 2011;40:418-23.

18. Shen K, Tang H, Jing R, Liu F, Zhou X. Application of triple-branched stent graft for Stanford type A aortic dissection: potential risks. Eur J Cardiothorac Surg. 2012;41:e12-7.

19. Sun L, Qi R, Zhu J, Liu Y, Chang Q, Zheng J. Repair of acute type A dissection: our experiences and results. Ann Thorac Surg. 2011;91:1147-52.

20. Sun LZ, Qi RD, Zhu JM, Liu YM, Zheng J. Total arch replacement combined with stented elephant trunk implantation: a new "standard" therapy for type A dissection involving repair of the aortic arch? Circulation. 2011;123:971-8.

Key Words: Total arch replacement, acute type A dissection, brachiocephalic vessels 